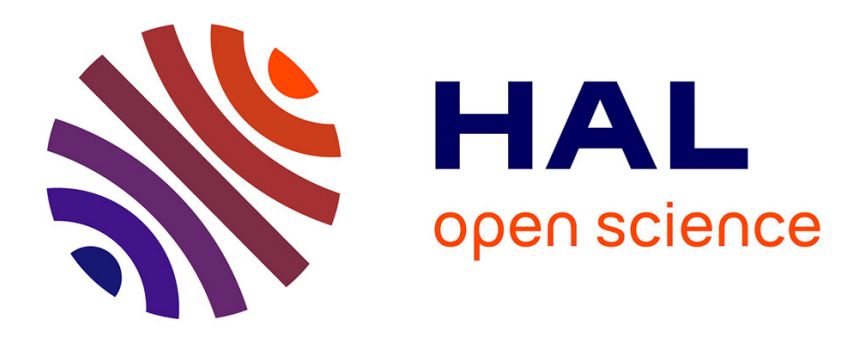

\title{
Apodization effects in Fourier transform infrared difference spectra
}

\author{
R.S. Bretzlaff, T.B. Bahder
}

\section{To cite this version:}

R.S. Bretzlaff, T.B. Bahder. Apodization effects in Fourier transform infrared difference spectra. Revue de Physique Appliquée, 1986, 21 (12), pp.833-844. 10.1051/rphysap:019860021012083300 . jpa-00245506

\section{HAL Id: jpa-00245506 https://hal.science/jpa-00245506}

Submitted on 1 Jan 1986

HAL is a multi-disciplinary open access archive for the deposit and dissemination of scientific research documents, whether they are published or not. The documents may come from teaching and research institutions in France or abroad, or from public or private research centers.
L'archive ouverte pluridisciplinaire HAL, est destinée au dépôt et à la diffusion de documents scientifiques de niveau recherche, publiés ou non, émanant des établissements d'enseignement et de recherche français ou étrangers, des laboratoires publics ou privés. 
Classification

Physics Abstracts

$07.65 \mathrm{G}$

\title{
Apodization effects in Fourier transform infrared difference spectra
}

\author{
R. S. Bretzlaff and T. B. Bahder $\left(^{+}\right)$ \\ Materials Sciences Laboratory, The Aerospace Corporation, El Segundo, California 90245, U.S.A.
}

(Reçu le 28 mai 1986, accepté le 26 août 1986)

\begin{abstract}
Résumé. - Dans le cas de bandes intenses des artefacts dus au processus d'apodisation peuvent apparaître dans les spectres infrarouges obtenus par transformée de Fourier. Ces effets ont déjà été étudiés sur des spectres obtenus par différence pour des fonctions d'apodisation de type boxcar ou triangulaire. Ce travail présente des simulations numériques réalisées avec une fonction d'apodisation de type Happ-Genzel couramment employée dans les instruments modernes. Pour comparer les performances des fonctions d'appareil de type boxcar, triangulaire, ou Happ-Genzel nous avons calculé (i) les artefacts apparaissant dans les spectres obtenus par différence; (ii) la variation de l'absorbance apparente au sommet d'un pic d'absorption en fonction de l'absorbance vraie ; (iii) une mesure intégrée de l'erreur due à ces artefacts pour une bande d'absorption à profil lorentzien. L'étude est faite pour des valeurs de $\rho$ (rapport de la largeur totale du pic à mi-hauteur à la résolution) qu'on rencontre couramment en spectroscopie de transmission d'échantillons solides ou translucides.
\end{abstract}

\begin{abstract}
Artifacts may occur in Fourier transform infrared (FTIR) spectra due to the apodization of the interferograms of intense bands. Selected examples of boxcar and triangular apodization effects on difference spectra have been previously reported. This paper reports the first such calculation performed for the HappGenzel apodization function, which is often used on modern spectrometers. In order to compare boxcar, triangular, and Happ-Genzel apodization functions we calculate (i) difference-spectrum artifacts, (ii) apparent versus true peak absorbances, and (iii) a measure of integrated artifact area for several true peak intensities of Lorentzian-band shapes. Values of $\rho$ (ratio of full bandwidth at half height to nominal resolution) are emphasized which commonly occur in the transmission-mode spectroscopy of transparent or translucent solid samples.
\end{abstract}

\section{Introduction.}

1.1 ORIGIN OF THE PROBLEM. - Fourier transform infrared (FTIR) analysis of aging in polymeric electrical insulation has been shown to be important in describing the dielectric breakdown process [1]. Continuing chemical reactions during aging cause variations in concentrations of functional groups. If physical rearrangements of polymer chains occur during aging, then there is also a change in the molecular environment, and hence in the effective dipole strength of the molecular oscillators. Such chemical and physical changes cause changes in a specimen's molecular vibration spectrum. The FTIR spectra taken before and after specimen aging reflect these changes, which are typically displayed as the digital subtraction of the initial spectrum from the final spectrum.

We are interested in extending the method of reference [1] to other insulations. However, it is not

$\left(^{+}\right)$Current address : Harry Diamond Laboratory, 2800 Powder Mill Road, Adelpli, MD 20783, U.S.A. apparent why this method works at all. In transmission-mode analysis, Mitsui, et al., used specimens 5$10 \mu$ thick, while we have so far analysed specimens 7-12 $\mu$ thick. Our unsubtracted infrared spectra have peak absorbances in the 2.0 to 3.0 range. Under the assumption of boxcar or triangular apodization (see below), it has been shown that the FTIR spectrometer behaves non linearly and gives large artifacts in the difference spectra. On the other hand, the data of Mitsui et al., as well as our own preliminary work, seem to be meaningful. We hypothesize therefore that the Happ-Genzel apodization function, which we used, has a very different mathematical effect on the computed infrared spectrum and, in particular, does not give rise to large artifacts. It is the objective of this paper to check this hypothesis by calculating and comparing the effects of boxcar, triangle, and Happ-Genzel apodization functions upon FTIR difference spectra.

1.2 ORIGIN AND IMPORTANCE OF APODIZATION FUNCTIONS. - Each data point in an interferogram contains some information about all the data points in the Fourier transformed spectrum. Real spectro- 
meters, with only a finite maximum optical path difference, do not utilize all of the true interferogram data points, which exist over an infinite range of optical path differences. Therefore, there is some degree of error in all of the spectral data points. Apodization is the attempt to make do with the available information by multiplying the collected interferogram by a given function before Fourier transformation. Outside of the data collection range, all apodization functions are defined to be identically equal to zero. Different apodization functions may be chosen for different purposes, such as suppressing side lobes or minimizing smearing of the central absorption peak. Rabolt and Bellar [2] give useful information on side-lobe magnitudes and centralpeak broadening obtained with three common apodizations, the boxcar, triangle, and Happ-Genzel functions, which are listed in detail in section 2.2 below.

Boxcar apodization arises naturally in a Michelson interferometer with finite mirror travel, because it multiplies collected interferogram data points by unity and is defined to be zero outside the range of mirror travel. One may ask, however, why triangular, Happ-Genzel, or any other function might be preferred in any particular case. Norton and Beer [3] analysed the «quality " of some 1150 different trigonometric and algebraic apodizing functions in terms of a Filler diagram. Relative lobe magnitude was plotted against relative central-peak half width. Each apodizing function is represented by one point on the Filler diagram. There appeared to be a boundary, to the left of, and below, which no function-point exists. Norton and Beer challenged the scientific community to formulate an existence proof for this mathematical boundary, to describe that boundary analyticaly, and to specify classes of apodization functions falling on that boundary. They noted that the commonly used triangular apodization was far from the apparent optimum line on the Filler diagram. The desired mathematical proofs have not appeared.

Meanwhile there have been significant advances in our knowledge of apodization effects in selected areas. Anderson and Griffiths [4] and Griffiths [5] demonstrated by calculation and experiment the effect of boxcar and triangular apodization on FTIR difference spectra. These results were based on a calculation procedure first defined in reference [6]. They showed that the apparent peak absorbance $A_{0}^{(\text {a })}$ was a function of the true peak absorbance $A_{0}{ }^{(\mathrm{t})}$, as well as $\rho_{\mathrm{G}}$ (ratio of nominal resolution to full bandwidth at half-height) and the particular instrumental line shape (ILS) function. The ILS function is the Fourier transform of the apodization function. They suggested that the basic requirement for good spectral subtraction is not photometric accuracy but merely a linear $A_{0}^{\left({ }^{(a)}\right.}$ versus $A_{0}^{(\mathrm{t})}$ response (for fixed $\rho_{\mathrm{G}}$ and ILS function), i.e., that Beer's law apply to the apparent bands in the absorbance range of interest. For sufficiently large $A_{0}{ }^{(\mathrm{t})}$, the response curves become nonlinear, and complete spectral compensation becomes impossible. Complete spectral compensation in this sense means the attainment of an identically zero difference spectrum from the scaled subtraction of spectra of two similar samples of different thickness. We refer to excursions from zero of such a perfectly scaled subtraction as spectral artifacts arising from apodization. Clearly, artifacts can be due to other sources (sample imperfections or electronic noise) which fall outside the scope of this discussion.

1.3 UNRESOLVED ISSUES IN APODIZATION. - Two problems present themselves: (1) In none of the extant literature is the effect of Happ-Genzel apodization on difference spectra calculated, even though this is one of the most common functions used in practice. The Happ-Genzel response curves, $A_{0}{ }^{(a)}$ versus $A_{0}^{(\mathrm{t})}$, for several $\rho$ values are similarly lacking. (2) Perfect spectral compensation is impossible under some conditions, such as for intense bands. However, a certain spectral artifact level may be tolerable in a given experiment. For example, the experimentally determined artifact levels in reference [7] were plotted on vertical display scales of zero \pm several hundreths of an absorbance unit, suggesting that experimental difference-spectral excursions much greater than about \pm 0.05 absorbance unit would not be interpreted as artifacts. Although a large number of calculated and experimental difference-spectra artifacts are available in references [4] and [5], there is no concise and comprehensive tabulation of artifact sizes as a function of $A_{0}^{(t)}, \rho\left(=1 / \rho_{\mathrm{G}}\right)$, and ILS function. Thus there is no ready reference for selecting an apodization function for difference-spectrum analysis and predicting the size of the corresponding artifacts. Such a reference is essential in order to gauge the adequacy, as opposed to the completeness, of spectral compensation.

The importance of these calculations may be judged from the following observations. Any deviation from Beer's law for the apparent peaks will result in finite difference spectra peaks. These peaks can derive from (1) adventitious factors such as are listed in references [8] and [9] and are generally known : beam polarization and sample nonuniformities such as non-planar faces and internal boundaries which reflect, refract, or scatter light out of the detector's field of view. In addition, (2), apodization artifacts originate when a finite spectrometer operates on samples whose thicknesses produce peak absorbances in a non-linear range of $A_{0}^{(a)}$ versus $A_{0}^{(\mathrm{t})}$. Finally, (3), if the first two sources can be shown to be small, then the remaining, genuine, apparent difference spectra peaks can be analysed as arising from changes in the true peaks. In this case the inherent sample absorption coefficient, $a$, thickness, $b$, or dipole concentration, $c$, must have changed in order to give the observed difference spectrum peak. Furthermore, if an appropriate scaling factor is chosen, thickness variations may be 
cancelled, and only changes in $a$ or $c$ need be considered.

Careful sample preparation may take care of problem (1). If the maximum apodization artifacts can be determined and shown to be small, problem (2) may be negligible, leaving real data to interpret. Therefore it is imperative to calculate difference spectral artifacts for several diferent $\rho$ and $A_{0}{ }^{(t)}$ values and to compare the results of boxcar, triangle, and Happ-Genzel apodization. A typical nominal resolution of $2 \mathrm{~cm}^{-1}$ is assumed. Bands of many solid samples fall between 10 and $50 \mathrm{~cm}^{-1}$ in full width at half height. Therefore, we consider $\rho=5$ and $25\left(\rho_{\mathrm{G}}=0.2\right.$ and 0.04$)$ as bracketing cases for typical solid-sample analysis. Several $A_{0}{ }^{(t)}$ values up to at least three absorbance units are considered.

\section{Theoretical definition of the problem.}

2.1 SPECTROMETER RESPONSE EQUATION. - We give a brief derivation of the spectrometer response equation. This equation relates the true spectrum to the apparent spectrum, which is the output from an ideal spectrometer and includes effects of apodization. The true intensity per unit wavenumber, $\bar{B}_{\mathrm{t}}(\nu)$, is the Fourier transform of $\bar{I}(\delta)$, the total intensity due to all wavenumbers when the path difference is $\delta$. We introduce the dimensionless variables $x=2 \pi \nu L, y=\delta / L$ and the functions $I(y)=\bar{I}(L y)$ and $B_{\mathrm{t}}(x)=\bar{B}_{\mathrm{t}}\left(\frac{x}{2 \pi L}\right)$, which satisfy :

$$
\begin{gathered}
B_{\mathrm{t}}(x)=L \int_{-\infty}^{\infty} I(y) e^{i x y} \mathrm{~d} y \\
I(y)=\frac{1}{2 \pi L} \int_{-\infty}^{\infty} B_{\mathrm{t}}(x) e^{-i x y} \mathrm{~d} x .
\end{gathered}
$$

Since data is collected over a finite range of mirror positions we introduce the apodization function $a(y)$, which is equal to zero for $|y|>1$. We identify the length $2 L$ as the total mirror travel distance and define the apparent spectrum $B_{\mathrm{a}}(x)$, which is an approximation to the true spectrum, by the equation :

$$
B_{\mathrm{a}}(x)=\frac{L}{M} \int_{-\infty}^{\infty} a(y) I(y) e^{i x y} \mathrm{~d} y,
$$

where $M$ is a constant. This constant is determined by requiring the integrated intensity of the apparent and true spectra to be equal. This calculation is facilitated by defining the Fourier pair :

$$
\begin{gathered}
\sigma(x)=L \int_{-\infty}^{\infty} a(y) e^{i x y} \mathrm{~d} y \\
a(y)=\frac{1}{2 \pi L} \int_{-\infty}^{\infty} \sigma(x) e^{-i x y} \mathrm{~d} x .
\end{gathered}
$$

Now

$$
\int_{-\infty}^{\infty} B_{\mathrm{t}}(x) \mathrm{d} x=\int_{-\infty}^{\infty} B_{\mathrm{a}}(x) \mathrm{d} x
$$

leads to $M=a(0)$. Thus, equation (3) becomes :

$$
B_{\mathrm{a}}(x)=\frac{L \int_{-\infty}^{\infty} a(y) I(y) e^{i x y} \mathrm{~d} y}{\frac{1}{2 \pi L} \int_{-\infty}^{\infty} \sigma\left(x^{\prime}\right) \mathrm{d} x^{\prime}} .
$$

Using equation (5), equation (7) can be rewritten as :

$$
B_{\mathrm{a}}(x)=\frac{\int_{-\infty}^{\infty} \mathrm{d} x^{\prime \prime} B_{\mathrm{t}}\left(x^{\prime \prime}\right) \sigma\left(x^{\prime \prime}-x\right)}{\int_{-\infty}^{\infty} \sigma\left(x^{\prime}\right) \mathrm{d} x^{\prime}}
$$

The true transmittance $T^{(t)}(x)$, is the ratio of the true intensity, $B_{\mathrm{t}}(x)$, to the spectral distribution of the infrared beam, $J(x)$. The apparent transmittance $T^{(\mathrm{a})}(x)$ is the ratio of the apparent spectral intensity $B_{\mathrm{a}}(x)$ with the sample in place to the apparent reference intensity when the sample is out of the beam. Thus

$$
\begin{aligned}
& T^{(\mathrm{a})}(x)= \\
& =\frac{\int_{-\infty}^{\infty} \mathrm{d} x^{\prime} T^{(\mathrm{t})}\left(x^{\prime}\right) J\left(x^{\prime}\right) \sigma\left(x^{\prime}-x\right)}{\int_{-\infty}^{\infty} \mathrm{d} x^{\prime} J\left(x^{\prime}\right) \sigma\left(x^{\prime}-x\right)} .
\end{aligned}
$$

Under certain conditions (see Appendix 1) the apparent and true transmittances in equation (9) can be related by the spectrometer response equation :

$$
\begin{aligned}
T^{(\mathrm{a})}(x) & = \\
& =\frac{\int_{0}^{\infty} \mathrm{d} x^{\prime} T^{(\mathrm{t})}\left(x^{\prime}\right) \sigma\left(x^{\prime}-x\right)}{\int_{0}^{\infty} \mathrm{d} x^{\prime} \sigma\left(x^{\prime}-x\right)} .
\end{aligned}
$$

Equation (10) is a standard result often quoted $[4,5$, $6,10]$. The true and apparent transmittance and absorbance are related by :

$$
\begin{aligned}
& T^{(\mathrm{t})}(x)=10^{-A^{(\mathrm{t})}(x)} \\
& T^{(\mathrm{a})}(x)=10^{-A^{(\mathrm{a})}}(x) .
\end{aligned}
$$

In all our calculations we choose a Lorentzian line shape for the true absorbance

$$
A^{(t)}(x)=\frac{A_{0}^{(t)}}{\left(\frac{x-x_{0}}{\tilde{\rho}}\right)^{2}+1}
$$


where $\tilde{\rho}=\rho \pi, \rho=2 \gamma L$, and $\gamma$ is the half width at half height, in wave-numbers, of the band. We calculate the apparent transmittance and absorbance from the true functions, using equations (10), (11) and (12) and compile a comprehensive set of difference-spectral artifacts for bands of typical solid samples.

2.2 ILS FUNCTIONS. - In the following equations we list the three apodization functions and their respective ILS functions which we use in our calculations, see equation (4). We append a subscript $i=1$, 2,3 to $a_{i}(x)$ and $\sigma_{i}(x)$ to distinguish among the three apodization functions. The apodization functions, $a_{i}(y)$, depend on the dimensionless length, $y$, which is the optical path difference divided by the maximum optical path difference, $L$. The ILS functions, $\sigma_{i}(x)$, depend on the dimensionless frequency, $x=2 \pi \nu L$, where $\nu$ is the frequency in wavenumbers.

Boxcar :

$$
\begin{aligned}
& a_{1}(y)= \begin{cases}1 & |y|<1 \\
0 & \text { otherwise }\end{cases} \\
& \sigma_{1}(x)=2 L \frac{\sin x}{x} .
\end{aligned}
$$

Triangle :

$$
\begin{aligned}
& a_{2}(y)= \begin{cases}1-|y| & |y|<1 \\
0 & \text { otherwise }\end{cases} \\
& \sigma_{2}(x)=\frac{L \sin ^{2}(x / 2)}{(x / 2)^{2}} .
\end{aligned}
$$

\section{Happ-Genzel :}

$$
\begin{gathered}
a_{3}(y)= \begin{cases}c_{1}+c_{2} \cos (\pi y) & |y|<1 \\
0 & \text { otherwise }\end{cases} \\
\sigma_{3}(x)= \\
=2 L\left[c_{1} \frac{\sin x}{x}+\frac{c_{2}}{2}\left\{\frac{\sin x}{\pi-x}-\frac{\sin x}{\pi+x}\right\}\right] .
\end{gathered}
$$

In equations (15a)-(15b), $c_{1}$ and $c_{2}$ are constants. It should be noted that an extra factor of two was written in the argument of the cosine in the HappGenzel function in reference [2], (see Ref. [11]).

2.3 LIMITING CASES. - When the true absorbance is weak, $A^{(\mathrm{t})}(x) \ll 1$ for all $x$, and we can evaluate equation (10) by expanding the exponential function :

$$
\begin{aligned}
& T^{(t)}(x)=10^{-A^{(t)}}(x)= \\
& =1-A^{(t)}(x) \ln 10+\ldots .
\end{aligned}
$$

Using this in equation (11) leads to :

$$
\begin{aligned}
T^{(a)}(x) & \cong 1-\ln (10) \times \\
& \times \frac{\int_{0}^{\infty} \mathrm{d} x^{\prime} A^{(t)}\left(x^{\prime}\right) \sigma\left(x^{\prime}-x\right)}{\int_{0}^{\infty} \mathrm{d} x^{\prime} \sigma\left(x^{\prime}-x\right)},
\end{aligned}
$$

where we have kept only the first two terms in equation (16a). Taking the negative of the base ten logarithm of equation (17), we have :

$$
\begin{aligned}
A^{(\mathrm{a})}(x) & \cong \\
\cong & \frac{\int_{0}^{\infty} \mathrm{d} x^{\prime} A^{(\mathrm{t})}\left(x^{\prime}\right) \sigma\left(x^{\prime}-x\right)}{\int_{0}^{\infty} \mathrm{d} x^{\prime} \sigma\left(x^{\prime}-x\right)},
\end{aligned}
$$

where use is made of $\log _{10}(1-y)=$ $\ln (1-y) / \ln 10$ and $\ln (1-y) \approx-y$ for $y \ll 1$, with $y$ being the right hand side of equation (17). Equation (17) shows that the response $A^{(a)}(x)$ (the apparent absorbance) depends linearly on the true absorbance $A^{(t)}(x)$. For a Lorentzian band, given in equation (12), we see that the apparent absorbance $A^{\text {(a) }}(x)$ scales linearly with the true peak absorbance $A_{0}{ }^{(t)}$. This was observed in a numerical calculation (Refs. [4-6]).

In the limit of strong absorbance the true transmission is essentially zero over some bandwidth $=2 \alpha$, and we then write :

$$
T^{(t)}(x)=\left\{\begin{array}{rr}
1 & 0<x<x_{0}-\alpha \\
0 & x_{0}-\alpha<x<x_{0}+\alpha \\
1 & x_{0}+\alpha<x<\infty .
\end{array}\right.
$$

Using this in equation (10) leads to :

$$
\begin{aligned}
& T_{i}^{(\mathrm{a})}(x)= \\
& =1-\frac{\sum_{i}\left(x_{0}-x-\alpha\right)-\sum_{i}\left(x_{0}-x+\alpha\right)}{\sum_{i}(-x)-\sum_{i}(\infty)},
\end{aligned}
$$

where we have introduced the indefinite integral:

$$
\sum_{i}(x)=\int \sigma_{i}(x) \mathrm{d} x .
$$

For the case of box car apodization, $i=1$, equation (20) can be evaluated to be :

$$
\begin{aligned}
& T_{1}^{(\mathrm{a})}(x)= \\
& =1-\frac{\mathrm{si}\left(x_{0}-x+\alpha\right)-\mathrm{si}\left(x_{0}-x-\alpha\right)}{\pi+\operatorname{si}(x)},
\end{aligned}
$$


where the sine integral function is defined as [12] :

$$
\operatorname{si}(z)=-\frac{\pi}{2}+\int_{0}^{z} \frac{\sin t}{t} \mathrm{~d} t .
$$

In all applications $x \gg 1$, so si $(x)$ in the denominator of equation (22) is close to zero. Since $x-x_{0}$ and $\alpha$ are independent parameters we see that $T^{(a)}(x)$ can achieve unphysical negative values. This is purely a consequence of apodization applied to intense bands. Similar results can be derived for triangle and Happ-Genzel apodizations.

\section{Numerical calculation.}

Values of $\rho$ were chosen to bracket our range of interest in analysing solid polymer samples. The full width at half height (FWHM) of infrared absorbance bands in solid polymer samples is typically between $10 \mathrm{~cm}^{-1}$ and $50 \mathrm{~cm}^{-1}$. A common nominal resolution is $2 \mathrm{~cm}^{-1}$. Therefore $\rho$ values between 5 and $25\left(\rho_{\mathrm{G}}\right.$ between 0.2 and 0.04 ) were analysed. In addition, $\rho=1$ results are shown for comparison with some previous results of Griffiths [5] and Anderson and Griffiths $[4,6]$. For calculating difference spectra, $A_{0}{ }^{(t)}$ was chosen to be $1.3,2.0$, and 3.0. The maximum true absorbance of the reference-spectrum for subtraction was chosen to be 1.1 in all cases. Several hundred data points were calculated between $x_{0}-5 \pi \rho$ and $x_{0}+5 \pi \rho$. As in reference [5], the criterion for subtraction was chosen to be :

$$
\begin{aligned}
\Delta A(x) & = \\
= & A^{(\mathrm{a})}(x)-\left(\frac{A_{0}^{(\mathrm{t})}}{A_{0, \mathrm{ref}}^{(\mathrm{t})}}\right) A_{\mathrm{ref}}^{(\mathrm{a})}(x) .
\end{aligned}
$$

Hence, the factors for scaled subtraction were 1.18 , 1.82 , and 2.73. It should be noted that the graphs of $\Delta A(x)$ are plotted versus $x-x_{0}$ in all figures. Also, the $\pm 5 \pi \rho$ range of $x$ corresponds to approximately $\pm 16, \pm 80$, and \pm 400 for $\rho=1,5$, and 25 , respectively. Finally appendix 2 shows why the criterion is reasonable. True peak heights and true baselines may be assumed to scale with thickness. Baselines (i.e. constant functions) are unaffected by the convolution in equation (10). Therefore, apparent baselines scale with thickness. Thus, apparent baselines scale with true peak heights, and equation (24) is equivalent to a spectral subtraction based on thickness.

For each type of apodization, we substitute for $\sigma(x)$ into equation (10) the functions $\sigma_{i}(x)$, given in equations (13b), (14b) and (15b). For each ILS function the denominator can be integrated directly. Each of the numerators are integrated by parts in order to avoid slowly convergent integrals. Let the numerator of the right side of equation (10) be $N_{i}(x)$ and the denominator be $D_{i}(x)$, where $i=1,2,3$ for boxcar, triangular, and Happ-Genzel functions, respectively. Using the definitions :

$$
\begin{gathered}
f\left(x-x_{0}\right)=A^{(\mathrm{t})}(x) \ln 10 \\
T_{i}^{(\mathrm{a})}=\frac{N_{i}(x)}{D_{i}(x)},
\end{gathered}
$$

we then have

\section{Boxcar :}

$$
\begin{gathered}
D_{1}(x)=\int_{0}^{\infty} \mathrm{d} x^{\prime} \frac{\sin \left(x-x^{\prime}\right)}{x-x^{\prime}}=\pi+\operatorname{si}(x) . \\
N_{1}(x)=\{\pi+\operatorname{si}(x)\} e^{-f\left(x_{0}\right)}+\int_{0}^{\infty} \mathrm{d} x^{\prime} \operatorname{si}\left(x^{\prime}-x\right) e^{-f\left(x^{\prime}-x_{0}\right)} \frac{\mathrm{d} f\left(x^{\prime}-x_{0}\right)}{\mathrm{d} x^{\prime}} . \\
T_{1}^{(\mathrm{a})}(x)=e^{-f\left(x_{0}\right)}+\frac{\int_{0}^{\infty} \mathrm{d} x^{\prime} \operatorname{si}\left(x^{\prime}-x\right) e^{-f\left(x^{\prime}-x_{0}\right)} \frac{\mathrm{d} f\left(x^{\prime}-x_{0}\right)}{\mathrm{d} x^{\prime}}}{\pi+\operatorname{si}(x)} .
\end{gathered}
$$

Triangle :

$$
D_{2}(x)=\operatorname{si}(2 x)+\pi-\frac{\sin ^{2} x}{x}
$$

$N_{2}(x)=D_{2}(x) e^{-f\left(x_{0}\right)}+$

$$
+\int_{0}^{\infty} \mathrm{d} x^{\prime}\left\{\operatorname{si}\left[2\left(x^{\prime}-x\right)\right]-\frac{\sin ^{2}\left(x^{\prime}-x\right)}{x^{\prime}-x}\right\} e^{-f\left(x^{\prime}-x_{0}\right)} \frac{\mathrm{d} f\left(x^{\prime}-x_{0}\right)}{\mathrm{d} x^{\prime}} .
$$


Thus,

$$
\begin{aligned}
T_{2}^{(\mathrm{a})}(x) & = \\
& =e^{-f\left(x_{0}\right)}+\frac{\int_{0}^{\infty} \mathrm{d} x^{\prime}\left\{\mathrm{si}\left[2\left(x^{\prime}-x\right)\right]-\frac{\sin ^{2}\left(x^{\prime}-x\right)}{x^{\prime}-x}\right\} e^{-f\left(x^{\prime}-x_{0}\right)} \frac{\mathrm{d} f\left(x^{\prime}-x_{0}\right)}{\mathrm{d} x^{\prime}}}{\pi+\operatorname{si}(2 x)-\frac{\sin ^{2} x}{x}} .
\end{aligned}
$$

Happ-Genzel :

$$
\begin{aligned}
& D_{3}(x)=c_{1}[\pi+\operatorname{si}(x)]+\frac{c_{2}}{2}[2 \pi+\operatorname{si}(x-\pi)+\operatorname{si}(x+\pi)] . \\
& N_{3}(x)=e^{-f\left(x_{0}\right)} \cdot D_{3}(x)+\int_{0}^{\infty} \mathrm{d} x^{\prime} e^{-f\left(x^{\prime}-x_{0}\right)} \frac{\mathrm{d} f\left(x^{\prime}-x_{0}\right)}{\mathrm{d} x^{\prime}} \times \\
& \times\left\{c_{1} \operatorname{si}\left(x^{\prime}-x\right)+\frac{c_{2}}{2}\left(\operatorname{si}\left(x^{\prime}-x+\pi\right)+\operatorname{si}\left(x^{\prime}-x-\pi\right)\right)\right\} . \\
& T_{3}^{(\mathrm{a})}(x)=e^{-f\left(x_{0}\right)}+ \\
& +\frac{\int_{0}^{\infty} \mathrm{d} x^{\prime} e^{-f\left(x^{\prime}-x_{0}\right)} \frac{\mathrm{d} f\left(x^{\prime}-x_{0}\right)}{\mathrm{d} x^{\prime}}\left\{c_{1} \operatorname{si}\left(x^{\prime}-x\right)+\frac{1}{2} c_{2}\left(\operatorname{si}\left(x^{\prime}-x+\pi\right)+\operatorname{si}\left(x^{\prime}-x-\pi\right)\right)\right\}}{\pi+c_{1} \operatorname{si}(x)+\frac{c_{2}}{2}(\operatorname{si}(x-\pi)+\operatorname{si}(x+\pi))} .
\end{aligned}
$$

In all three cases when the integrated term is evaluated at $x^{\prime}=\infty$, it vanishes. Equations (27c), $(28 c)$, and $(29 c)$ were used in our numerical calculations. The presence of the term $\mathrm{d} f\left(x^{\prime}-x_{0}\right) / \mathrm{d} x^{\prime}$ in the integrals improves the rate of convergence. The sine integral function was evaluated piecewise, according to formulae 5.2.8, 5.2.10, 5.2.38, and 5.2.39 of reference [12].

\section{Results.}

The main purpose of this investigation is to assess the adequacy (not the completeness) of spectral compensation. To this end, difference-spectral artifacts were calculated and cataloged in figure 1 $(\rho=1)$, figure $2 \quad(\rho=5)$, and figure 3 $(\rho=25)$. In the figures 1-3 the notation BX, TG, and HG are used for boxcar, triangular, and HappGenzel apodization, respectively. The values of $A_{0}{ }^{(t)}$ were selected to be $1.3,2.0$, and 3.0. The reference spectrum for subtraction has $A_{0}{ }^{(t)}=1.1$ in each case.

In figures 1-3, consider first the difference-spectral artifacts arising from boxcar apodization. Substantial positive-going artifacts exist for $\rho=1$, in agreement with the previous result in reference [5]. The band for $A_{0}^{(t)}=3.0$ cannot be calculated at $\rho=1$, because the apparent transmittance has become negative. For $\rho=5$ and $A_{0}{ }^{(t)}=1.3$ and 2.0, the artifacts are two orders of magnitude smaller in amplitude, at about 0.0005 and 0.008 , respectively. For $\rho=5$ and $A_{0}{ }^{(t)}=3.0$, the maximum artifact amplitude is about 0.1 absorbance unit. For $\rho=25$, the envelopes inside which the artifacts exist are about $0.002,0.01$, and 0.10 absorbance unit in maximum amplitude for $A_{0}^{\left({ }^{(t)}\right.}=1.3,2.0$, and 3.0, respectively. (A detailed view of the oscillations within these envelopes is provided below.) Thus we conclude that experimental difference-spectra peaks much greater than 0.1 absorbance unit in boxcar apodized spectra obtained for $\rho$ between 5 and 25 and for sample/reference spectrum intensities of up to 3.0/1.1 cannot be attributed to apodization of Lorentzian absorbance bands. Difference-spectra peaks on the order of 0.1 absorbance unit or less might be attributable to apodization effects under these conditions.

In figures 1-3, consider next the difference-spectral artifacts for triangular apodization. Relatively large, negative-going artifacts up to 0.8 absorbance unit in amplitude arise for $\rho=1$. The results are not very much better for $\rho=5$. (The result for $\rho=5$ $\left(\rho_{\mathrm{G}}=0.2\right)$ and $A_{0}{ }^{(t)}=1.3$ is in agreement with that in Ref. [5].) For $\rho=25$ we again obtain envelopes within which the artifacts exist and which 


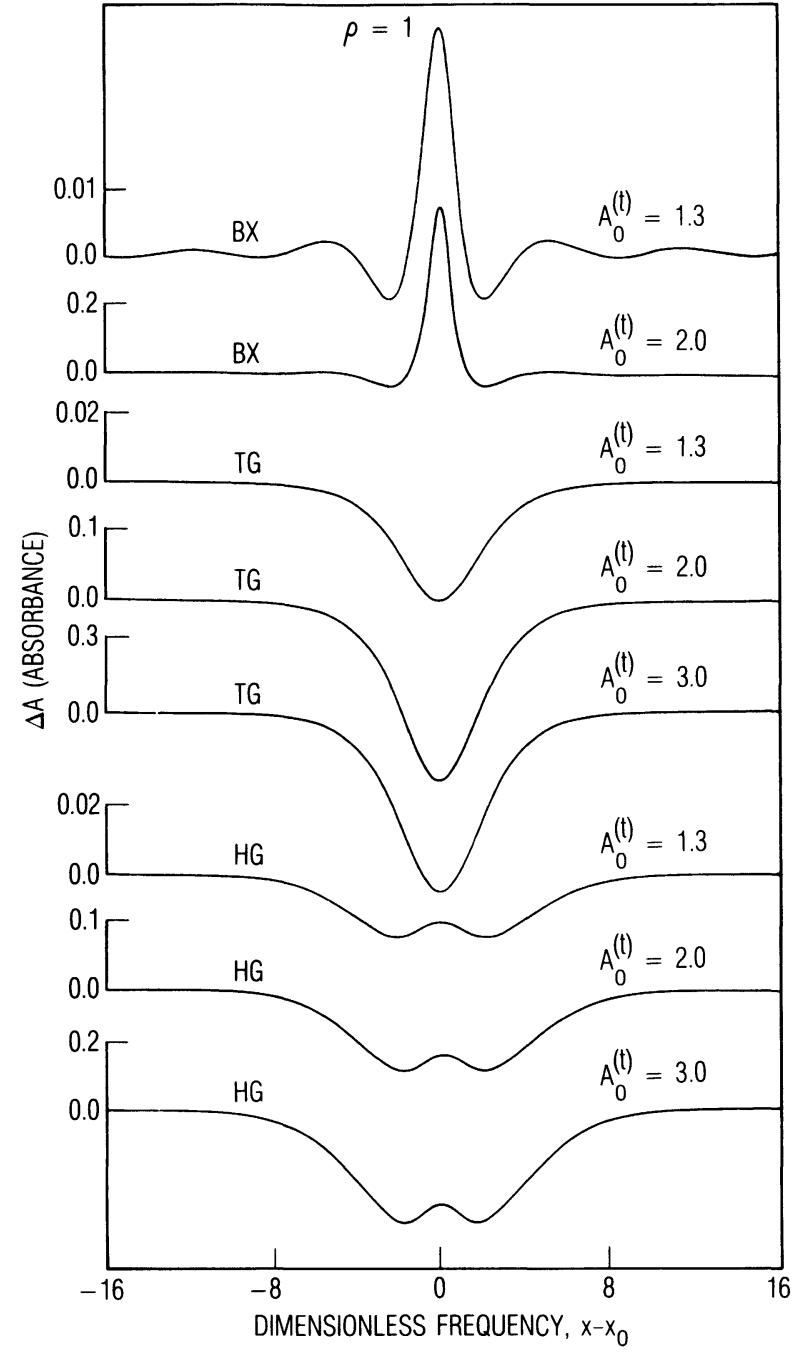

Fig. 1. - Difference-spectra artifacts, $\Delta A$, are graphed versus the dimensionless frequency, $x-x_{0}$, relative to the true peak position, $x_{0}$, for the case of $\rho=1$.

are 0.002 and 0.06 in amplitude for $A_{0}^{(\mathrm{t})}=1.3$ and 2.0 , respectively. At $A_{0}{ }^{(t)}=3.0$ the artifact amplitude is again large, at 0.4 absorbance unit. Thus, we conclude that experimental difference spectra of intense, triangularly apodized spectra obtained for $\rho$ between 5 and 25 would have to be significantly greater than 0.8 absorbance unit to avoid the possibility of being spectral artifacts.

In figures 1-3, consider finally the difference-spectral artifacts for Happ-Genzel apodization. The artifact amplitudes for $\rho=1$ are again sizeable, but not quite as large as in the preceding two cases. It is interesting to note that Happ-Genzel apodization leads to double-negative-lobed artifacts, which are quite different from the previous cases. For $\rho=5$, the artifact amplitudes are $0.002,0.015$, and 0.05 for $A_{0}{ }^{(t)}=1.3,2.0$, and 3.0, respectively. The first two of these numbers are slightly worse than for the boxcar function, but the last number is only half as large as for the boxcar function. Both boxcar and

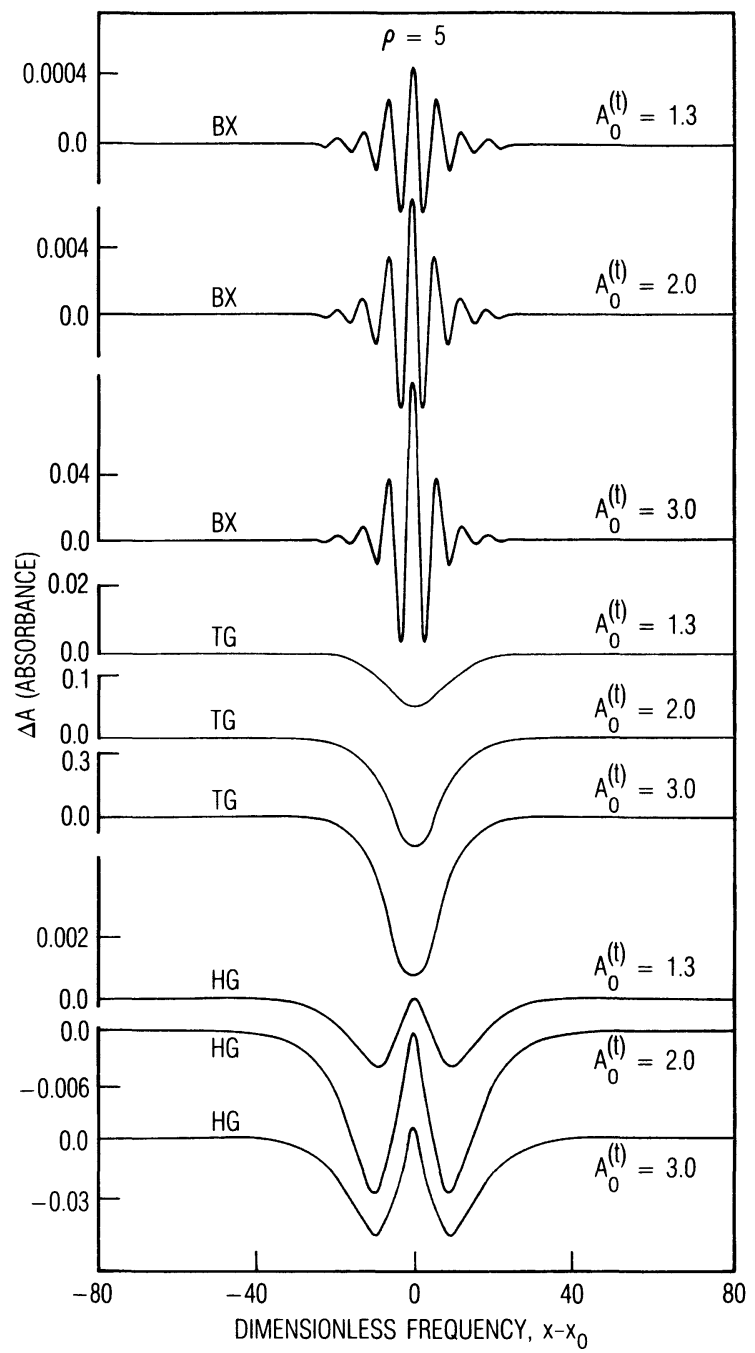

Fig. 2. - Difference-spectra artifacts, $\Delta A$, are graphed versus the dimensionless frequency, $x-x_{0}$, relative to the true peak position, $x_{0}$, for the case of $\rho=5$.

Happ-Genzel apodizations are clearly superior to triangular apodization. It is at $\rho=25$, however, that Happ-Genzel apodization is clearly seen to be the best of the three functions studied. Here, the maximum amplitude of the artifact envelope $\left(A_{0}{ }^{(t)}=3.0\right)$ is seen to be 0.02 . Thus, we conclude that experimental difference spectra peaks much greater than 0.05 absorbance unit in Happ-Genzel apodized spectra obtained for $\rho$ between 5 and 25 and for sample/reference sample intensities of up to 3.0/1.1 can not be attributed to apodization of Lorentzian bands. Difference-spectra peaks on the order of 0.05 or less might be attributable to apodization effects.

The data of figures 1-3 are summarized in table I. Details of the oscillations occurring in some of the $\rho=25$ difference spectra are shown in figure 4 .

We discuss the deviation of $A^{(a)}(x)$ from $A^{(t)}(x)$ in terms of two distinct error criteria. First, a local measure of error which relates the 


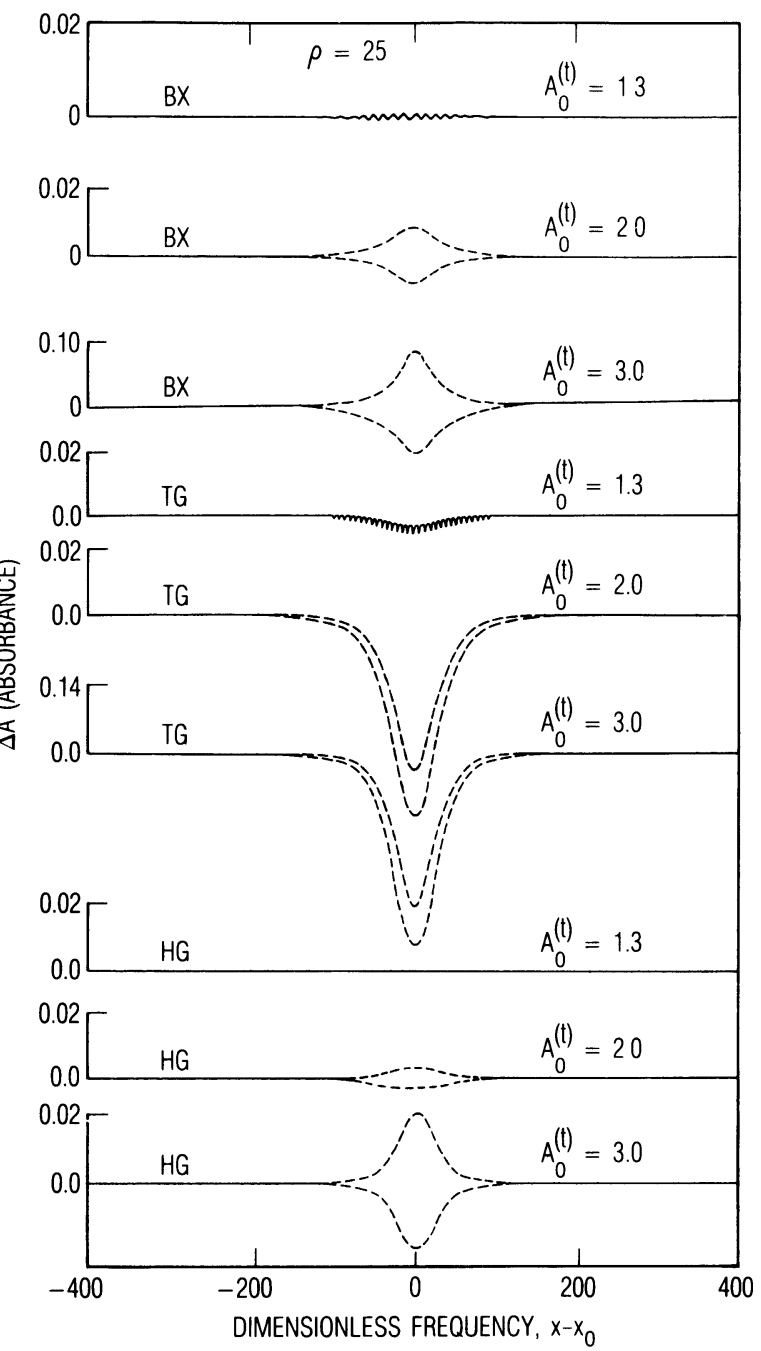

Fig. 3. - Difference-spectra artifacts, $\Delta A$, are graphed versus the dimensionless frequency, $x-x_{0}$, relative to the true peak position, $x_{0}$, for the case of $\rho=25$.

differences in true and apparent peak intensities (barring phase shifting) is provided by the graph of $A_{0}^{(\mathrm{a})}=A^{(\mathrm{a})}\left(x_{0}\right)$ versus $A_{0}^{(\mathrm{t})}=A^{(\mathrm{t})}\left(x_{0}\right)$, where $x_{0}$ is the dimensionless peak frequency. For small $A_{0}^{(\mathrm{t})}$ and all apodizations the $A_{0}^{(\mathrm{a})}$ versus $A^{(\mathrm{t})}$ graph is linear, see equation (18). Deviations occurring for larger $A_{0}^{(t)}$ are perhaps better discussed in terms of a second distributed error criteria. A distributed measure of error, $E$, versus $A_{0}^{\left({ }^{(t)}\right.}$, is defined by :

$$
E=\int_{0}^{\infty}\left\{A^{(\mathrm{a})}(x)-A^{(\mathrm{t})}(x)\right\}^{2} \mathrm{~d} x .
$$

The $E$ criterion is in some cases superior, because the rapid oscillations (compared to the bandwidth) seen in some of the $\rho=25$ difference spectra can result in phase-shifting (of $A^{(a)}(x)$ relative to

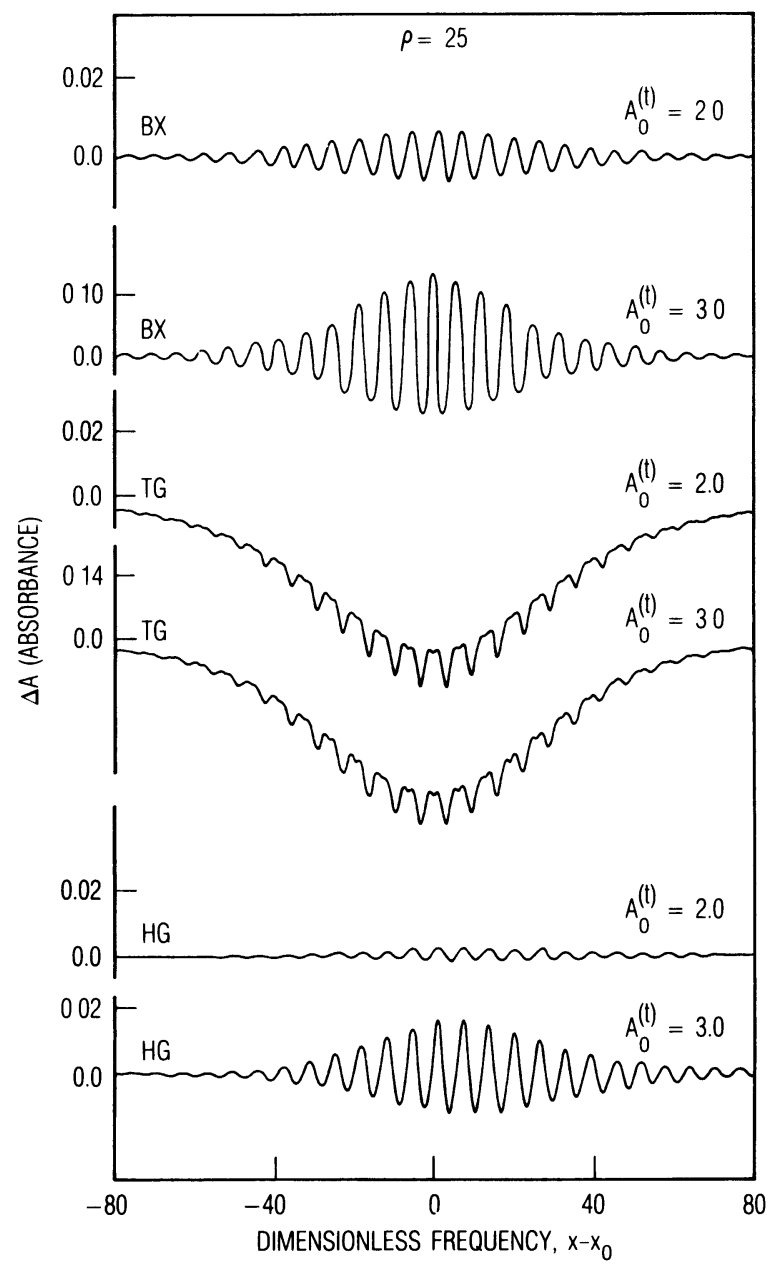

Fig. 4. - Details of the difference-spectra artifacts from figure 3 are graphed versus the dimensionelss frequency, $x-x_{0}$, relative to the true peak position, $x_{0}$.

$\left.A^{(\mathrm{t})}(x)\right)$ and apparently linear $A_{0}^{\left({ }^{(a)}\right.}$ versus $A_{0}{ }^{\left({ }^{t}\right)}$ graphs even though $E$ is large.

Now we turn our attention to figure 5 , which are the $A_{0}^{\left({ }^{(a)}\right.}$ versus $A_{0}{ }^{\left({ }^{(}\right)}$graphs for boxcar-apodized spectra. As has been previously pointed out in references [4] and [5], the boxcar $A_{0}^{(\mathrm{a})}\left(A_{0}^{(\mathrm{t})}\right)$ function represents a nearly linear response for $\rho=5$ or 25 for $A_{0}{ }^{(t)} \leq 3.0$. Positive deviations occur for smaller $\rho$ values, as has been seen in the preceding difference spectra. The boxcar $E$ versus $A_{0}{ }^{(t)}$ results are shown in figure 6. There is a rapid increase in $E$ for $A_{0}{ }^{(t)}$ above $2.5,3.0$, and 1.5 absorbance unit for $\rho=25,5$, and 1 , respectively.

In figure 7 the $A_{0}{ }^{(a)}$ versus $A_{0}{ }^{(t)}$ graph is shown for the case of triangular apodization. In agreement with references [4] and [5] there is a large deviation from linearity at modest $A_{0}{ }^{(t)}$ values. Similarly, the 
Table I.

\begin{tabular}{|c|c|c|c|c|}
\hline \multirow[b]{2}{*}{ Figure } & \multicolumn{4}{|c|}{$\begin{array}{c}\text { Maximum artifact sizes arising from apodization of a Lorentzian } \\
\text { band with various ILS, } \rho \text {, and } A_{0}^{\left({ }^{(t)}\right.}\left({ }^{1}\right)\end{array}$} \\
\hline & Apod Fn & $\rho$ & $A_{0}^{(\mathrm{t})}($ Sample $)$ & Max Artifact Size $\left(^{2}\right)$ \\
\hline 1 & 1 (Boxcar) & 1 & 1.3 & +0.03 \\
\hline 1 & 1 & 1 & 2.0 & +0.50 \\
\hline 一 & 1 & 1 & 3.0 & (diverges) \\
\hline 2 & 1 & 5 & 1.3 & \pm 0.0004 \\
\hline 2 & 1 & 5 & 2.0 & \pm 0.008 \\
\hline 2 & 1 & 5 & 3.0 & \pm 0.09 \\
\hline 3 & 1 & 25 & 1.3 & $< \pm 0.002$ \\
\hline 3 & 1 & 25 & 2.0 & \pm 0.01 \\
\hline 3 & 1 & 25 & 3.0 & \pm 0.10 \\
\hline 1 & 2 (Triangle) & 1 & 1.3 & -0.03 \\
\hline 1 & 2 & 1 & 2.0 & -0.25 \\
\hline 1 & 2 & 1 & 3.0 & -0.80 \\
\hline 2 & 2 & 5 & 1.3 & -0.015 \\
\hline 2 & 2 & 5 & 2.0 & -0.15 \\
\hline 2 & 2 & 5 & 3.0 & -0.80 \\
\hline 3 & 2 & 25 & 1.3 & -0.002 \\
\hline 3 & 2 & 25 & 2.0 & -0.06 \\
\hline 3 & 2 & 25 & 3.0 & -0.40 \\
\hline 1 & 3 (Happ-Genzel) & 1 & 1.3 & -+-0.02 \\
\hline 1 & 3 & 1 & 2.0 & -+-0.10 \\
\hline 1 & 3 & 1 & 3.0 & -+-0.30 \\
\hline 2 & 3 & 5 & 1.3 & -+-0.002 \\
\hline 2 & 3 & 5 & 2.0 & -+-0.015 \\
\hline 2 & 3 & 5 & 3.0 & -+-0.05 \\
\hline 3 & 3 & 25 & 1.3 & $< \pm 0.002$ \\
\hline 3 & 3 & 25 & 2.0 & \pm 0.002 \\
\hline 3 & 3 & 25 & 3.0 & \pm 0.02 \\
\hline
\end{tabular}

( $\left.{ }^{1}\right) A_{0, \text { ref }}^{(\mathrm{t})}=1.1$.

$\left({ }^{2}\right)$ Signs indicate characteristic artifact behaviour : positive for boxcar, negative for triangle, double negative lobes for Happ-Genzel, « \pm » for apparent rapid oscillations.

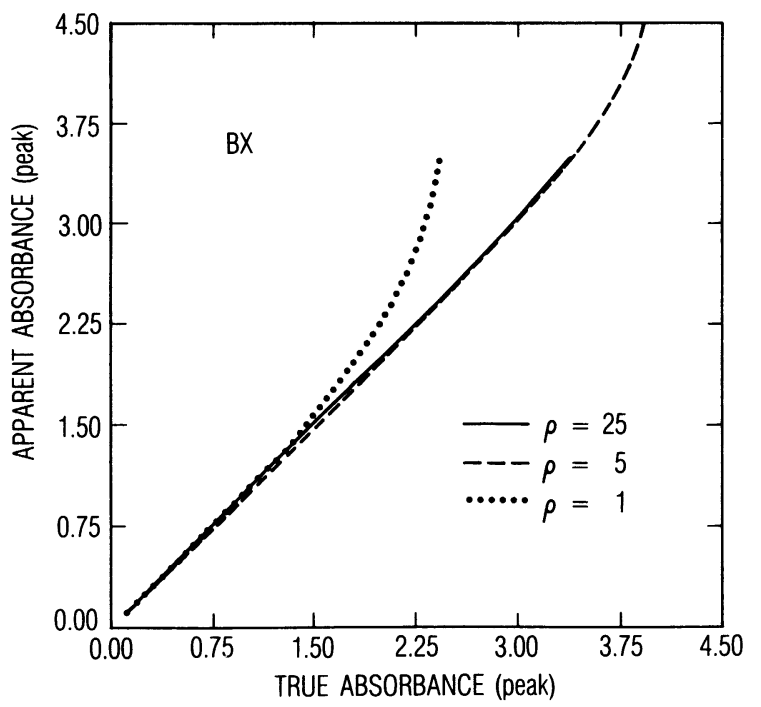

Fig. 5. - The apparent peak absorbance of boxcar-apodized bands is graphed versus the true peak absorbance for $\rho=1,5$, and 25 .

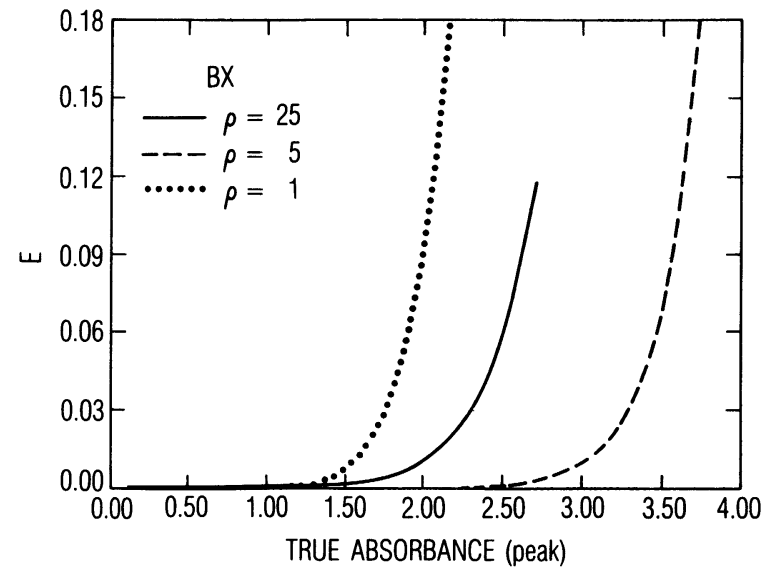

Fig. 6. - The integrated error, $E$, for boxcar apodized bands is graphed versus the true peak absorbance for $\rho=1,5$, and 25 . 


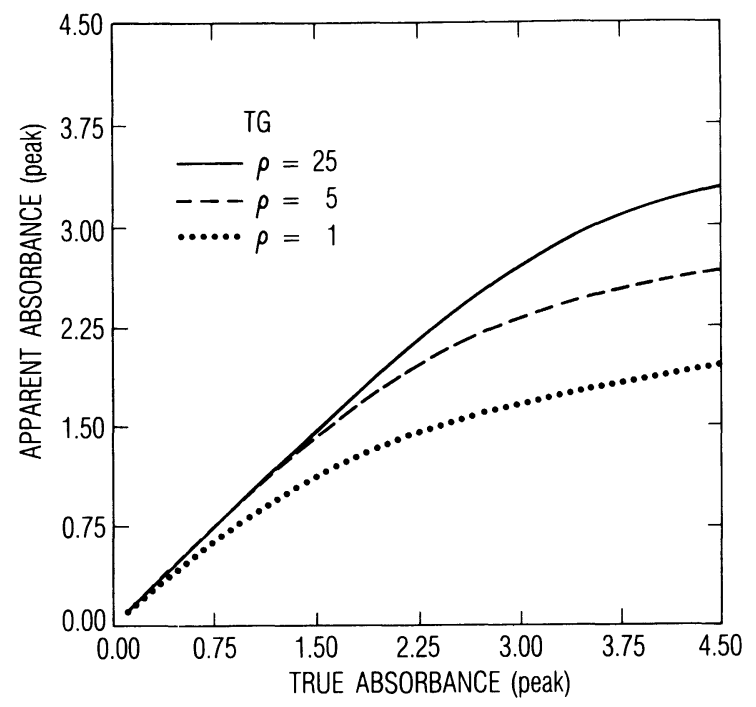

Fig. 7. - The apparent peak absorbance of triangularly apodized bands is graphed versus the true peak absorbance for $\rho=1,5$, and 25 .

quantity, $E$, (shown in Fig. 8) increases rapidly after $A_{0}{ }^{(t)}=1.5,1.0$, and 0.5 for $\rho=25,5$, and 1 , respectively.

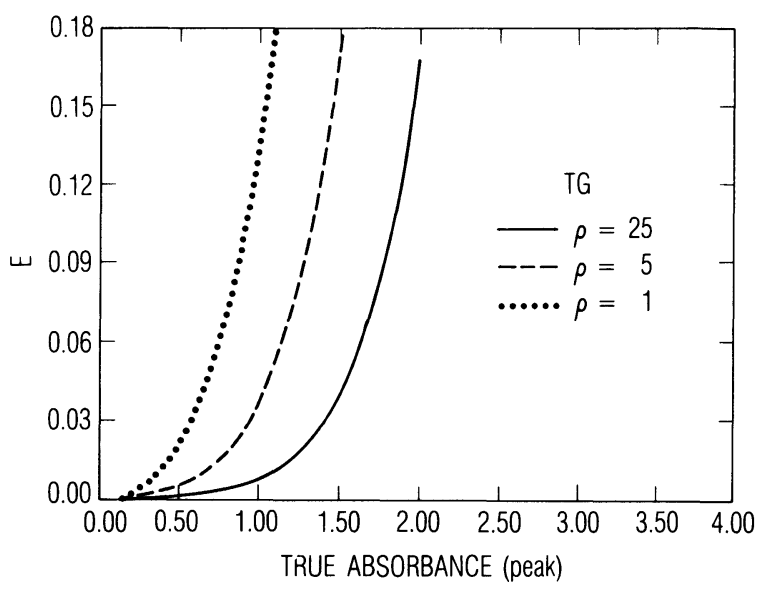

Fig. 8. - The integrated error, $E$, for triangularly apodized bands is graphed versus the true peak absorbance for $\rho=1,5$, and 25 .

Finally, the excellent linearity of the $A_{0}^{\left({ }^{(a)}\right.}$ versus $A_{0}^{\left({ }^{(}\right)}$graph for Happ-Genzel apodization for $\rho=25$ and 5 is shown in figure 9 . There is a slightly nonlinear curve for $\rho=1$, but as noted, the spectroscopy of (condensed-phase) polymers is almost always carried out between $\rho=5$ and 25 . In figure 10 , the quantity, $E$, is seen to greatly increase above $A_{0}{ }^{(t)}=3.0,2.0$, and 0.5 for $\rho=25,5$, and 1 , respectively.

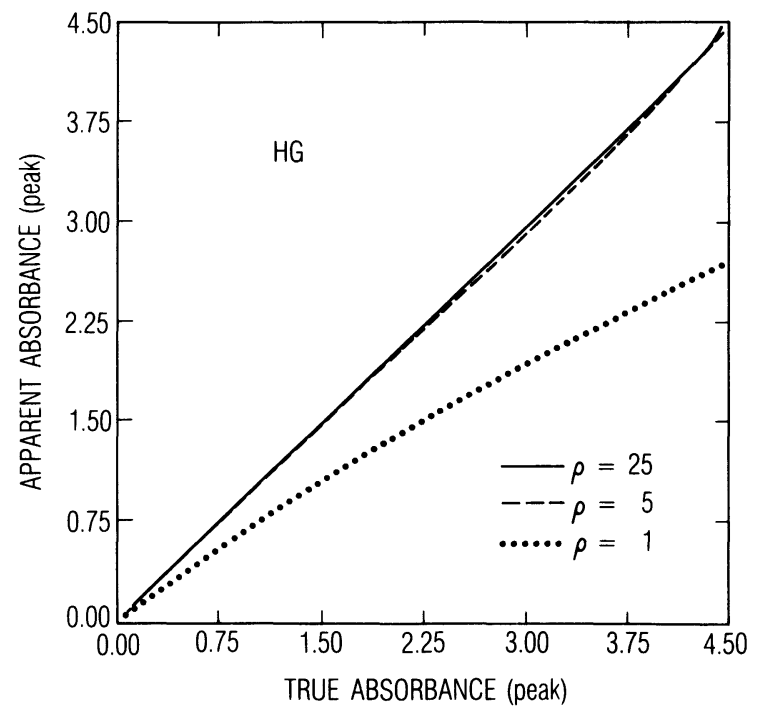

Fig. 9. - The apparent peak absorbance of Happ-Genzelapodized bands is graphed versus the true peak absorbance for $\rho=1,5$, and 25 .

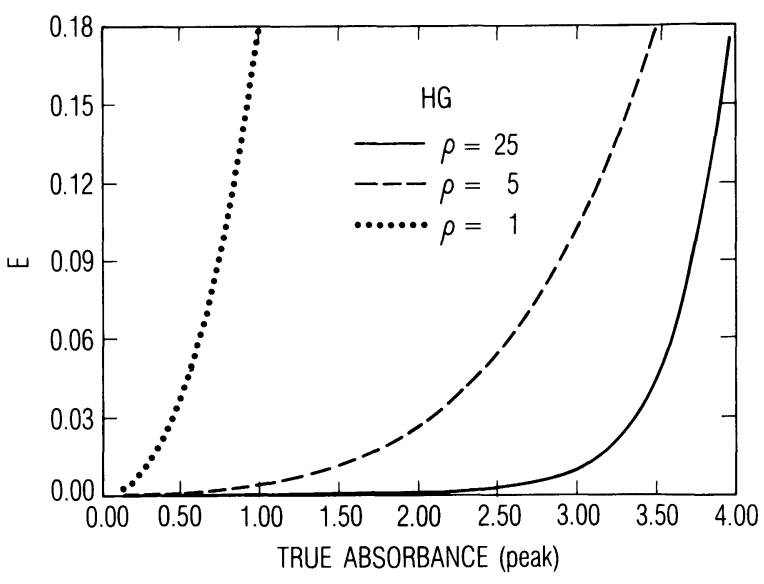

Fig. 10. - The integrated error, $E$, is graphed versus the true peak absorbance for $\rho=1,5$, and 25 .

\section{Summary.}

Difference spectrum analysis is an important tool in the transmission-mode infrared spectroscopy of transparent or translucent solid samples, but such a technique cannot be used indiscriminantly. Adventitious artifacts may appear from beam polarization and sample nonuniformities such as non-planar faces and internal boundaries which reflect, refract, or scatter light out of the detector's field of view. These pitfalls must be carefully avoided on a case by case basis. Then it must be considered what artifacts are introduced by the measurement process, which has been the topic of this paper. We have used Lorentzian absorbance bands to determine spectral artifacts, but we expect qualitatively similar results for Gaussian or combination Gaussian-Lorentzian bands. 
It was shown that triangular apodization of Lorentzian bands leads to a high degree of « unsubtractibility ", where up to 0.80 absorbance unit artifact amplitudes can be introduced for typical solid samples $(\rho=5$ to $\rho=25)$. These negativegoing artifacts manifest themselves as nonlinear $A_{0}^{\left({ }^{(a)}\right.}$ versus $A_{0}{ }^{(t)}$ curves which fall distinctly below the (photometrically accurate) $45^{\circ}$ slope. The quantity $E$, which is related to the total area of the spectral artifacts, rapidly increases for $A_{0}{ }^{(t)}$ above $1.5,1.0$, and 0.5 absorbance unit for $\rho=25,5$, and 1 , respectively.

Boxcar apodization is better than triangular apodization for difference spectrum analysis, giving at most a 0.10 artifact amplitude $(\rho=5$ to $\rho=25)$. These artifacts manifest themselves as nonlinear $A_{0}^{\left({ }^{(a)}\right.}$ versus $A_{0}^{\left({ }^{(}\right)}$curves which rise above the (photometrically accurate) $45^{\circ}$ slope. The quantity, $E$, rapidly increases for $A_{0}{ }^{(t)}$ above $2.5,3.0$, and 1.5 absorbance unit for $\rho=25,5$, and 1 , respectively.

Happ-Genzel apodization is the best of the three functions investigated giving a maximum artifact size of $0.05(\rho=5$ to $\rho=25)$. These artifacts manifest themselves as $A_{0}^{\left({ }^{(a)}\right.}$ versus $A_{0}^{\left({ }^{(t)}\right.}$ curves which lie close to the $45^{\circ}$ slope for $\rho=25$ to 5 . In contrast, the $\rho=1$ result is slightly nonlinear and decidedly below the $45^{\circ}$ slope. Moreover, in order for any subtraction band to be attributable to Happ-Genzel apodization (apart from the rapid oscillations common to the apodization of all intense bands) a characteristic double lobed difference band must appear. This follows from the "triple boxcar " nature of Happ-Genzel apodization (see Eq. (15b)). Thus there can be in principle no objection, on the grounds of Happ-Genzel apodization effects, to the evaluation of difference spectral bands much greater than 0.05 as being real data. Furthermore, this is true for subtractions of bands of intensity 1.1 from bands of intensity 3 . As long as one is operating on bands with $\rho$ between 5 and 25 , the small 0.05 « unsubtractibility » due to Happ-Genzel apodization is likely to blend in with all the other experimental factors to give a small residuum of error in the zero baseline of the difference spectra.

\section{Acknowledgment}

This work was prepared under the auspices of the Aerospace Corporation's Aerospace Sponsored Research. The availability of the Space Sciences Laboratory « Dirac » VAX is gratefully acknowledged.

\section{Appendix 1}

SIMPLIFICATION OF THE SPECTROSCOPIC RESPONSE EQUATION. - In equation (9) note that the lower limits can be set equal to zero, because $J\left(x^{\prime}\right)$ is zero for $x<0$. Furthermore, for an absorption band with halfwidth $\gamma$ centred at wavenumber $\nu_{0}$, let $x_{0}=2 \pi \nu_{0} L$ and $\rho=2 \gamma L$ be the dimensionless position and characteristic width of the band. Let $x_{\mathrm{sw}}=2 \pi L\left(\nu_{\max }-\nu_{\min }\right)$ be the dimensionless spectral width of $J(x)$ where $\nu_{\max }$ and $\nu_{\min }$ are the band maximum and minimum wavenumbers. We can simplify the denominator of equation (9) as follows. The function $\sigma\left(x^{\prime}-x\right)$ is peaked at $x^{\prime}-x$ and has a characteristic width of the central peak which is of the order of unity. On the other hand the spectral width, $x_{\mathrm{sw}}$, of $J(x)$ has a characteristic width $x_{\mathrm{sw}} \gg 1$. Furthermore, $J\left(x^{\prime}\right)$ varies slowly with $x^{\prime}$. For this reason we can find a positive constant, $\Delta$, which can be used to break up the $x^{\prime}$ integration into three regions,

$$
\begin{gathered}
\text { I }: 0<x^{\prime}<x-\Delta, \\
\text { II }: x-\Delta<x^{\prime}<x+\Delta, \\
\text { III }: x+\Delta<x^{\prime}<\infty,
\end{gathered}
$$

and which satisfies the condition $1 \ll \Delta \ll x_{\text {sw }}$. In the denominator of equation (9) the integrals in regions I and III are negligible in comparison with the region II integral. Making a variable change $t=-x^{\prime}+x$ in region I of integration yields :

$$
\int_{\Delta}^{x} J(x-t) \sigma(t) \mathrm{d} t,
$$

which is small because $J(y) \rightarrow 0$ for $y \rightarrow 0$ and because $\sigma(t)$ is small for $\Delta \gg 1$. Making the variable change $t=x^{\prime}-x$ in the integration of region III yields :

$$
\int_{\Delta}^{\infty} J(x+t) \sigma(t) \mathrm{d} t,
$$

which is again small because $J(y) \rightarrow 0$ and $\sigma(y) \rightarrow 0$ for $y \rightarrow \infty$. In region II one can expand $J\left(x^{\prime}\right)=J(x)+J^{\prime}(x)\left(x-x^{\prime}\right)$ since $J\left(x^{\prime}\right)$ has been assumed to be slowly varying. In this region $J^{\prime}(x)$ is of the order of $J(x) / x_{\mathrm{sw}}$ so that :

$$
J\left(x^{\prime}\right) \cong J(x)\left[1+0\left(\frac{1}{x_{\mathrm{sw}}}\right)\right] .
$$

Substituting these results into the denominator of equation (9), we have :

$$
\begin{aligned}
& J(x) \int_{x-\Delta}^{x+\Delta} \sigma\left(x^{\prime}-x\right) \mathrm{d} x^{\prime} \cong \\
& \cong J(x) \int_{0}^{\infty} \sigma\left(x^{\prime}-x\right) \mathrm{d} x^{\prime}
\end{aligned}
$$

where we have extended the limits and keep only the leading term. Using the same arguments for the numerator in equation (9) we can cancel the spectral function $J(x)$ in the numerator and denominator to arrive at equation 10. 


\section{Appendix 2}

BASELINE EFFECTS IN DIFFERENCE SPECTRA. Here we show that including a constant baseline proportional in magnitude to the peak heights does not affect the difference spectra. This is important to note, because some experimental procedures derive their scaling factors from the ratio of baseline magnitudes. Consider the apparent absorbances, $\bar{A}_{1}^{(\text {a })}(x)$ and $\bar{A}_{2}^{\left({ }^{a}\right)}(x)$ of two bands :

$$
\begin{aligned}
& \bar{A}_{1}^{(\mathrm{a})}(x)=d_{1}+A_{1}^{(\mathrm{a})}(x) \\
& \bar{A}_{2}^{(\mathrm{a})}(x)=d_{2}+A_{2}^{(\mathrm{a})}(x),
\end{aligned}
$$

where $A_{i}{ }^{(\text {a })}(x)$ are the Lorentzians, and $d_{i}$ are the constant baseline absorbances $(i=1,2)$. Letting the true Lorentzian peak absorbances be $A_{0, i}^{(\mathrm{t})}$, we assume that the baselines $d_{i}=c \cdot A_{0, i}$ where $c$ is a constant. The factor $F$ for scaled subtraction could be taken as :

$$
\begin{aligned}
F=\frac{\bar{A}_{1}^{(\mathrm{a})}(x \rightarrow 0)}{A_{2}^{(\mathrm{a})}(x \rightarrow 0)} & = \\
& =\frac{c \cdot A_{0,1}^{(\mathrm{t})}+0}{c \cdot A_{0,2}^{(\mathrm{t})}+0}=\frac{A_{0,1}^{(\mathrm{t})}}{A_{0,2}^{(\mathrm{t})}},
\end{aligned}
$$

where $x \rightarrow 0$ refers to a wing region. Now the difference spectrum is :

$$
\begin{aligned}
\bar{A}_{1}^{(\mathrm{a})}( & (x)-F \cdot \bar{A}_{2}^{(\mathrm{a})}(x)= \\
= & c \cdot A_{0,1}^{(\mathrm{t})}+A_{1}^{(\mathrm{a})}(x) \\
& -\frac{A_{0,1}^{(\mathrm{t})}}{A_{0,2}^{(\mathrm{t})}}\left[c \cdot A_{0,2}^{(\mathrm{t})}+A_{2}^{(\mathrm{a})}(x)\right] \\
& =A_{1}^{(\mathrm{a})}(x)-\frac{A_{0,1}^{(\mathrm{t})}}{A_{0,2}^{(\mathrm{t})}} A_{2}^{(\mathrm{a})}(x),
\end{aligned}
$$

which is the criterion used in the text and in reference [5]. This shows that baselines introduced in this fashion do not affect the artifacts in the difference spectra.

\section{References}

[1] Mitsui, H., Yoshimitsu, T., Mizutani, Y., UmeмOTо, K., IEEE Trans. Elect. Insul. EI 16 (1981) 533 .

[2] Rabolt, J. F., Bellar, R., Appl. Spectr. 35 (1981) 132.

[3] Norton, R. H., Beer, R., J. Opt. Soc. Am. 66 (1976) 259.

[4] Anderson, R. J., Griffiths, P. R., Analyt. Chem. 50 (1978) 1804.

[5] GriffithS, P. R., Appl. Spect. 31 (1977) 497.

[6] ANDERSON, R. J., GRIFFITHS, P. R., Analyt. Chem. 47 (1975) 2339.

[7] Strassburger, J., Smith, I. T., Appl. Spectr. 33 (1979) 283.
[8] Gillette, P. C., Koenig, J. L., Appl. Spectr. 38 (1984) 334.

[9] Hirschfeld, T., Cody, C., Appl. Spectr. 31 (1977) 551.

[10] Seshadri, K. S., Jones, R. N., Spectrochim. Acta 19 (1963) 1013.

[11] Bertie, J. E., Apodization and Phase Correction, in J. R. Durig (ed.) Analytical Applications of FTIR to Molecular and Biological Systems, NATO Advanced Study Series, (1980).

[12] Abramovitz, M., Stegun, I. A., (eds.), Handbook of Mathematical Functions (Dover) 1972. 